DOI: $10.21105 /$ joss.03095

\title{
IGRINS RV: A Python Package for Precision Radial Velocities with Near-Infrared Spectra
}

\section{Software}

- Review ¿

- Repository ¿

- Archive ¿

Editor: Dan Foreman-Mackey ¿ד Reviewers:

- @jmbrewer

- Ogully

Submitted: 01 February 2021 Published: 10 June 2021

\section{License}

Authors of papers retain copyright and release the work under a Creative Commons Attribution 4.0 International License (CC BY 4.0).

In partnership with

\section{A A AMERICAN AMERICAN
ASTRONOMICAL SOCIETY}

This article and software are linked with research article DOI 10.3847/1538-3881/abf5e7, published in the Astronomical Journal.

\section{Shih-Yun Tang ${ }^{1,2}$, Asa G. Stahl ${ }^{3}$, Christopher M. Johns-Krull ${ }^{3}$, L. Prato $^{1,2}$, and Joe Llama ${ }^{1}$}

1 Lowell Observatory, 1400 W. Mars Hill Road, Flagstaff, AZ 86001, USA 2 Department of Astronomy and Planetary Sciences, Northern Arizona University, Flagstaff, AZ 86011, USA 3 Department of Physics and Astronomy, Rice University, 6100 Main Street, Houston, TX 77005, USA

\section{Summary}

The relative radial velocity of a star with respect to the Sun can be calculated from its electromagnetic spectrum using the Doppler Effect. This line-of-sight motion, called the Radial Velocity (RV), is an essential tool for astrophysicists. RVs are not only used to detect and characterize exoplanets, but also play a key role in studies of binary stars, star clusters, and moving group member identification.

In the past decade, RVs have primarily been measured from spectra in the optical wavelength regime. This is partly because of advancements in detector technology, but also because of the paucity of Earth's atmospheric absorption features (telluric lines) in the optical. Yet for fainter, cooler, smaller stellar object like M-type stars (stars with mass less than half of the Sun), which emit more energy in the Near-Infrared (NIR), observations in the NIR can save a considerable amount of exposure time. Also, M-type stars are the most common type of star. This along with its size increases the detectability of Earth-like planets around them. Moreover, the stellar activity that can drive false positive exoplanet detections, e.g., star spots carried into view by stellar rotation, is shown to be less severe in the NIR compared to optical.

\section{Statement of need}

Current RV pipelines and techniques that can deliver RV precision in tens of $\mathrm{m} / \mathrm{s}$ (or better) in the NIR, e.g., PySHELL (Cale et al., 2019), wobble (Bedell et al., 2019), SERVAL (Zechmeister et al., 2018) or the PCA-based cross-correlating method used for the SPIRou spectrograph (Moutou et al., 2020), all require instruments that are highly stabilized and have well-characterized wavelength solutions. For example, the iSHELL spectrograph can be equipped with the methane isotopologue gas cell, and the SPIRou and the CARMENES (NIR channel) spectrographs come with uranium-neon hollow-cathode lamps and stabilized Fabry-Perot etalons. The Immersion GRating INfrared Spectrometer (IGRINS) spectrograph (G. Mace et al., 2016; Gregory Mace et al., 2018; Park et al., 2014; Yuk et al., 2010), on the other hand, was not designed to be RV-stable and comes with no means of wavelength calibration accurate enough to achieve RVs precise to tens of $\mathrm{m} / \mathrm{s}$ using existing techniques. A new approach to extract precision RVs is needed for an echelle spectrograph like IGRINS, which offers fertile ground for $R V$ science with its high resolution $(R \sim 45,000)$ and broad spectral grasp (the full $\mathrm{H}$ and $\mathrm{K}$ bands).

IGRINS RV is a pipeline tailored for extracting precision RVs from spectra taken with IGRINS on different facilities. This pipeline is built on the forward-modeling methodology that was 
successfully applied to CSHELL and PHOENIX spectra (Crockett et al., 2012) that utilized telluric lines as a common-path wavelength calibrator. Compared to RVs obtained by crosscorrelation with stellar templates adopted by past studies, IGRINS RV gives three times higher RV precision, about 25-30 m/s, around narrow-line stars in both $\mathrm{H}$ and $\mathrm{K}$ bands, shown by years of monitoring on two RV standard stars, GJ 281 and HD 26257. IGRINS RV also pushes this technique, using telluric lines as wavelength calibrator for RV calculations, to its limits as studies found the stability of the telluric lines is about 10-20 m/s (Figueira et al., 2010; Seifahrt \& Käufl, 2008). Moreover, IGRINS RV is also tailored to take into account specific aspects of the IGRINS instrument, like the variations in spectral resolution across the detector and the year-long $\mathrm{K}$ band detector defocus.

IGRINS RV has demonstrated its effectiveness in identifying orbiting companions by successfully recovering the planet-induced RV signals of HD 189733 and Tau Boo A. IGRINS RV lets users choose to obtain absolute RVs or relative RVs, depending on whether their priority is coarse RV characterization or more precise RV monitoring. The code extends the science capabilities of an already powerful spectrograph, which lacked a publicly available RV pipeline until now. It facilitates the detection and/or characterization of exoplanets, binary stars, star clusters, and moving group members, and it enables such studies to be done in a more precise and uniform way.

IGRINS RV makes use of the astropy (Astropy Collaboration et al., 2018, 2013) on handing sky coordinates and barycentric velocity correction, scipy (Virtanen et al., 2020) and numpy (Harris et al., 2020) on mathmatical calculation, nlopt (Box, 1965; Johnson, 2008) on the optimization process, pandas (McKinney, 2010; team, 2020) on data management, and matplotlib (Hunter, 2007) on plotting. We also used a part of code from BMC (Duarte \& Watanabe, 2021) for peak detection. IGRINS RV requires that the igrins plp v2.2.0 (Lee et al., 2017) and Telfit (Gullikson et al., 2014) packages be pre-installed. Detailed documentation and tutorials can be found on the GitHub wiki page.

\section{Acknowledgements}

Partial support for this work was provided by NASA Exoplanet Research Program grant 80NSSC19K-0289 to L. Prato. CMJ would like to acknowledge partial support for this work from grants to Rice University provided by NASA (award 80-NSSC18K-0828) and the NSF (awards AST-2009197 and AST-1461918). We are grateful for the generous donations of John and Ginger Giovale, the BF Foundation, and others which made the IGRINS-DCT program possible. Additional funding was provided by the Mt. Cuba Astronomical Foundation and the Orr Family Foundation. IGRINS was developed under a collaboration between the University of Texas at Austin and the Korea Astronomy and Space Science Institute (KASI) with the financial support of the US National Science Foundation under grants AST-1229522 and AST-1702267 to the University of Texas at Austin, and of the Korean GMT Project at KASI.

\section{References}

Astropy Collaboration, Price-Whelan, A. M., Sipőcz, B. M., Günther, H. M., Lim, P. L., Crawford, S. M., Conseil, S., Shupe, D. L., Craig, M. W., Dencheva, N., Ginsburg, A., Vand erPlas, J. T., Bradley, L. D., Pérez-Suárez, D., de Val-Borro, M., Aldcroft, T. L., Cruz, K. L., Robitaille, T. P., Tollerud, E. J., ... Astropy Contributors. (2018). The Astropy Project: Building an Open-science Project and Status of the v2.0 Core Package. Astronomical Journal, 156(3), 123. https://doi.org/10.3847/1538-3881/aabc4f

Astropy Collaboration, Robitaille, T. P., Tollerud, E. J., Greenfield, P., Droettboom, M., Bray, E., Aldcroft, T., Davis, M., Ginsburg, A., Price-Whelan, A. M., Kerzendorf, W. E., Conley,

Tang et al., (2021). IGRINS RV: A Python Package for Precision Radial Velocities with Near-Infrared Spectra. Journal of Open Source Software, 2 6(62), 3095. https://doi.org/10.21105/joss.03095 
A., Crighton, N., Barbary, K., Muna, D., Ferguson, H., Grollier, F., Parikh, M. M., Nair, P. H., ... Streicher, O. (2013). Astropy: A community Python package for astronomy. Astronomy \& Astrophysics, 558, A33. https://doi.org/10.1051/0004-6361/201322068

Bedell, M., Hogg, D. W., Foreman-Mackey, D., Montet, B. T., \& Luger, R. (2019). WOBBLE: A Data-driven Analysis Technique for Time-series Stellar Spectra. Astronomical Journal, 158(4), 164. https://doi.org/10.3847/1538-3881/ab40a7

Box, M. (1965). A new method of constrained optimization and a comparison with other methods. The Computer Journal, 8(1), 42-52. https://doi.org/10.1093/comjnl/8.1.42

Cale, B., Plavchan, P., LeBrun, D., Gagné, J., Gao, P., Tanner, A., Beichman, C., Xuesong Wang, S., Gaidos, E., Teske, J., Ciardi, D., Vasisht, G., Kane, S. R., \& von Braun, K. (2019). Precise Radial Velocities of Cool Low-mass Stars with iSHELL. Astronomical Journal, 158(5), 170. https://doi.org/10.3847/1538-3881/ab3b0f

Crockett, C. J., Mahmud, N. I., Prato, L., Johns-Krull, C. M., Jaffe, D. T., Hartigan, P. M., \& Beichman, C. A. (2012). A Search for Giant Planet Companions to T Tauri Stars. Astrophysical Journal, 761, 164. https://doi.org/10.1088/0004-637X/761/2/164

Duarte, M., \& Watanabe, R. N. (2021). Notes on Scientific Computing for Biomechanics and Motor Control (Version v0.0.2). Zenodo. https://doi.org/10.5281/zenodo.4599319

Figueira, P., Pepe, F., Lovis, C., \& Mayor, M. (2010). Evaluating the stability of atmospheric lines with HARPS. Astronomy \& Astrophysics, 515, A106. https://doi.org/10. 1051/0004-6361/201014005

Gullikson, K., Dodson-Robinson, S., \& Kraus, A. (2014). Correcting for Telluric Absorption: Methods, Case Studies, and Release of the TelFit Code. Astronomical Journal, 148(3), 53. https://doi.org/10.1088/0004-6256/148/3/53

Harris, C. R., Millman, K. J., Walt, S. J. van der, Gommers, R., Virtanen, P., Cournapeau, D., Wieser, E., Taylor, J., Berg, S., Smith, N. J., Kern, R., Picus, M., Hoyer, S., Kerkwijk, M. H. van, Brett, M., Haldane, A., Fernández del Río, J., Wiebe, M., Peterson, P., ... Oliphant, T. E. (2020). Array programming with NumPy. Nature, 585, 357-362. https://doi.org/10.1038/s41586-020-2649-2

Hunter, J. D. (2007). Matplotlib: A 2D graphics environment. Computing in Science \& Engineering, 9(3), 90-95. https://doi.org/10.1109/MCSE.2007.55

Johnson, S. G. (2008). The NLopt nonlinear-optimization package. In GitHub. https:// github.com/stevengj/nlopt

Lee, J.-J., Gullikson, K., \& Kaplan, K. (2017). Igrins/plp 2.2.0. Zenodo. https://doi.org/10. 5281 /zenodo. 845059

Mace, G., Kim, H., Jaffe, D. T., Park, C., Lee, J.-J., Kaplan, K., Yu, Y. S., Yuk, I.-S., Chun, M.-Y., Pak, S., Kim, K.-M., Lee, J.-E., Sneden, C. A., Afsar, M., Pavel, M. D., Lee, H., Oh, H., Jeong, U., Park, S., ... Park, B.-G. (2016). 300 nights of science with IGRINS at McDonald Observatory. Ground-Based and Airborne Instrumentation for Astronomy VI, 9908, 99080C. https://doi.org/10.1117/12.2232780

Mace, Gregory, Sokal, K., Lee, J.-J., Oh, H., Park, C., Lee, H., Good, J., MacQueen, P., Oh, J. S., Kaplan, K., Kidder, B., Chun, M.-Y., Yuk, I.-S., Jeong, U., Pak, S., Kim, K.-M., Nah, J., Lee, S., Yu, Y.-S., ... Jaffe, D. T. (2018). IGRINS at the Discovery Channel Telescope and Gemini South. Proceedings of the SPIE, 10702, 107020Q. https: //doi.org/10.1117/12.2312345

McKinney, Wes. (2010). Data Structures for Statistical Computing in Python. In Stéfan van der Walt \& Jarrod Millman (Eds.), Proceedings of the 9th Python in Science Conference (pp. 56-61). https://doi.org/10.25080/Majora-92bf1922-00a 
Moutou, C., Dalal, S., Donati, J.-F., Martioli, E., Folsom, C. P., Artigau, É., Boisse, I., Bouchy, F., Carmona, A., Cook, N. J., Delfosse, X., Doyon, R., Fouqué, P., Gaisné, G., Hébrard, G., Hobson, M., Klein, B., Lecavelier des Etangs, A., \& Morin, J. (2020). Early science with SPIRou: near-infrared radial velocity and spectropolarimetry of the planethosting star HD 189733. Astronomy \& Astrophysics, 642, A72. https://doi.org/10.1051/ 0004-6361/202038108

Park, C., Jaffe, D. T., Yuk, I.-S., Chun, M.-Y., Pak, S., Kim, K.-M., Pavel, M., Lee, H., Oh, H., Jeong, U., Sim, C. K., Lee, H.-I., Nguyen Le, H. A., Strubhar, J., Gully-Santiago, M., Oh, J. S., Cha, S.-M., Moon, B., Park, K., ... Park, B.-G. (2014). Design and early performance of IGRINS (Immersion Grating Infrared Spectrometer). Ground-Based and Airborne Instrumentation for Astronomy v, 9147, 91471D. https://doi.org/10.1117/12. 2056431

Seifahrt, A., \& Käufl, H. U. (2008). High precision radial velocity measurements in the infrared. A first assessment of the RV stability of CRIRES. Astronomy \& Astrophysics, 491(3), 929-939. https://doi.org/10.1051/0004-6361:200810174

team, T. pandas development. (2020). Pandas-dev/pandas: pandas (latest) [Computer software]. Zenodo. https://doi.org/10.5281/zenodo.3509134

Virtanen, P., Gommers, R., Oliphant, T. E., Haberland, M., Reddy, T., Cournapeau, D., Burovski, E., Peterson, P., Weckesser, W., Bright, J., van der Walt, S. J., Brett, M., Wilson, J., Millman, K. J., Mayorov, N., Nelson, A. R. J., Jones, E., Kern, R., Larson, E., ... SciPy 1.0 Contributors. (2020). SciPy 1.0: Fundamental Algorithms for Scientific Computing in Python. Nature Methods, 17, 261-272. https://doi.org/10.1038/ s41592-019-0686-2

Yuk, I.-S., Jaffe, D. T., Barnes, S., Chun, M.-Y., Park, C., Lee, S., Lee, H., Wang, W., Park, K.-J., Pak, S., Strubhar, J., Deen, C., Oh, H., Seo, H., Pyo, T.-S., Park, W.-K., Lacy, J., Goertz, J., Rand, J., \& Gully-Santiago, M. (2010). Preliminary design of IGRINS (Immersion GRating INfrared Spectrograph). Proceedings of the SPIE, 7735, 77351M. https://doi.org/10.1117/12.856864

Zechmeister, M., Reiners, A., Amado, P. J., Azzaro, M., Bauer, F. F., Béjar, V. J. S., Caballero, J. A., Guenther, E. W., Hagen, H.-J., Jeffers, S. V., Kaminski, A., Kürster, M., Launhardt, R., Montes, D., Morales, J. C., Quirrenbach, A., Reffert, S., Ribas, I., Seifert, W., ... Wolthoff, V. (2018). Spectrum radial velocity analyser (SERVAL). High-precision radial velocities and two alternative spectral indicators. Astronomy \& Astrophysics, 609, A12. https://doi.org/10.1051/0004-6361/201731483 